\title{
Diversidad de avispas parasitoides de la familia Encyrtidae (Hymenoptera: Chalcidoidea) del bosque tropical caducifolio en San Buenaventura, Jalisco, México
}

\section{Diversity of parasitoid wasps of the family Encyrtidae (Hymenoptera: Chalcidoidea) in the tropical dry forest of San Buenaventura, Jalisco, Mexico}

\author{
Beatriz Rodríguez-Velez ${ }^{1 *}$, Santiago Zaragoza-Caballero ${ }^{1}$, Felipe A. Noguera ${ }^{2}$, Enrique González-Soriano ${ }^{1}$, \\ Enrique Ramírez-García ${ }^{2}$, Alicia Rodríguez-Palafox ${ }^{\dagger}$ y Ricardo Ayala ${ }^{2}$ \\ ${ }^{1}$ Departamento de Zoología, Instituto de Biología, Universidad Nacional Autónoma de México, Apartado postal 70-153, 04510 México, D.F., México. \\ ${ }^{2}$ Estación de Biología Chamela, Instituto de Biología, Universidad Nacional Autónoma de Mexico. Apartado postal 21, 48980 San Patricio, Jalisco, \\ México. \\ *Correspondencia: brodriguez@ibiologia.unam.mx
}

\begin{abstract}
Resumen. Se presentan los resultados del estudio de la fauna de avispas parasitoides de la familia Encyrtidae en el bosque tropical caducifolio de San Buenaventura, Jalisco, México. El estudio se llevó a cabo de noviembre de 1996 a octubre de 1997. La recolección se realizó con trampas Malaise durante 5 días de cada mes. Se registró un total de 61 especies, 30 géneros, 13 tribus y 2 subfamilias. El mayor número de géneros y especies fue de la subfamilia Encyrtinae, con 22 géneros y 44 especies, seguida por Tetracneminae con 8 géneros y 17 especies. El género con mayor número de especies fue Metaphycus con 11. Las especies recolectadas no fueron abundantes, y su riqueza y abundancia varió estacionalmente, registrándose los valores más altos durante la temporada de lluvias. La similitud de la fauna de San Buenaventura, Jalisco con la de Huautla, Morelos fue mayor que la encontrada en otras regiones previamente estudiadas de bosque tropical caducifolio (San Javier, Sonora y Huatulco, Oaxaca).
\end{abstract}

Palabras clave: riqueza, abundancia, fenología.

\begin{abstract}
Results of a faunistic study of the parasitoid wasps of the family Encyrtidae of the tropical dry forest of San Buenaventura, Jalisco, Mexico are presented. The study was carried out from November 1996 to October 1997. Collecting technique was Malaise trapping. Collections were carried out during 5 days of every month. A total of 61 species, 30 genera, 13 tribes and 2 subfamilies were recorded. The subfamily with the largest number of species was Encyrtinae with 22 genera and 44 species, followed by Tetracneminae with 8 genera and 17 species. The genus with the largest number of species was Metaphycus with 11. Species had low abundance. Species richness and abundance varied with time, with the highest values recorded in the rainy season. The fauna of San Buenaventura, Jalisco was most similar to that of Huautla, Morelos, than to that of San Javier, Sonora and Huatulco, Oaxaca, all of them previously studied.
\end{abstract}

Key words: richness, abundance, phenology.

\section{Introducción}

La diversidad de himenópteros parasitoides en las regiones tropicales es generalmente más alta que en cualquier otra región (Wolda, 1983; Noyes, 1989). Sin embargo, las regiones tropicales son las menos conocidas y las que más rápidamente se están destruyendo (Wilson, 1988). El bosque tropical caducifolio (BTC) es uno de los ecosistemas más afectados, con casi la mitad de sus hábitats remplazados por tierras de cultivo y un incremento

Recibido: 17 agosto 2010; aceptado: 15 octubre 2010 †Fallecida muy acelerado en los cambios de sus hábitats y clima. Este tipo de bosques presenta un creciente deterioro a causa de las especies invasoras, así como por la persistente explotación de sus recursos y rápido aumento de la contaminación (Millennium Ecosystem Assessment, 2005). De acuerdo con datos de la International Naturist Federation (INF), la superficie del bosque tropical caducifolio en México es de $148130 \mathrm{~km}^{2}$, que corresponde al 7.6\% del territorio nacional (Trejo, 2010). A diferencia de las comunidades tropicales húmedas, este bosque tiene un gran número de endemismos (Ceballos y García, 1995). Desafortunadamente, esta comunidad es de las más amenazadas en 
el mundo (Janzen, 1988), por lo que el estudio de su biodiversidad es cada vez más urgente con el fin de generar información que contribuya a su conservación.

Los himenópteros parasitoides desempeñan un papel importante en casi todos los ecosistemas terrestres por ser endoparasitoides $\mathrm{y} / \mathrm{o}$ hiperparasitoides de otros artrópodos (Noyes, 1988). Su biología les da una gran importancia en las comunidades naturales, ya que ayudan a regular las poblaciones de muchas especies de insectos (Noyes, 1988). En particular, las avispas parasitoides de la familia Encyrtidae representan un grupo de microhimenópteros considerablemente diverso (Noyes, 1989).

Teniendo en cuenta este escenario, un grupo de investigadores del Instituto de Biología, UNAM inició un proyecto a largo plazo para entender la diversidad y patrones de distribución de los diversos grupos de insectos en el BTC en México. Como parte de este proyecto, se llevaron a cabo estudios en 8 regiones del país. Los encírtidos de 3 localidades (Huautla, Morelos, Rodríguez-Velez y Wolley, 2005; Huatulco, Oaxaca, Rodríguez-Velez et al., 2009; San Javier, Sonora, Rodríguez et al., en prensa) han sido analizados previamente.

En este trabajo se presenta el análisis de la diversidad de Encyrtidae del BTC de San Buenaventura, Jalisco, México y los datos obtenidos se comparan con las 3 regiones mencionadas, con el fin de proporcionar una mejor comprensión de la diversidad local y temporal del grupo examinado.

\section{Materiales y métodos}

Sitio de recolecta. La región de San Buenaventura, Jalisco, está localizada en la vertiente oriental de la sierra Cacoma de Manantlán (19॰45' 19" - $19^{\circ} 48^{\prime} 50^{\prime \prime} \mathrm{N}$ y $104^{\circ} 01^{\prime} 25^{\prime}$ " $104^{\circ} 08^{\prime} 25^{\prime \prime}$ O). De acuerdo con la clasificación de Köppen modificada por García (García, 1981), el clima es cálido subhúmedo de tipo Awo" (w)(i')g, con una precipitación promedio anual cercana a $747 \mathrm{~mm}$ durante el periodo de estudio, según la estación climatológica más cercana, Reservorio B. Badillo (CNA, 2000). La temperatura media para el mismo periodo fue de $23.8^{\circ} \mathrm{C}$, con temperaturas máxima y mínima de $31.9^{\circ} \mathrm{C}$ y $15.8^{\circ} \mathrm{C}$, respectivamente. La temperatura más alta se registró en mayo y la más baja en enero.

El BTC es el principal tipo de vegetación en el área de estudio. Las especies de árboles dominantes son Lysiloma acapulcense (Kunth) Benth. (Leguminosae), L. microphyllum Benth., Jacaratia mexicana A. DC. (Caricaceae), Amphypterigium adstringens (Schlecht.) (Julianiaceae), Entada polystachia (L.) DC en DC (Leguminosae), Ceiba aesculifolia (H. B. K.) Britt. et Baker (Bombacaceae), Acacia macilenta Rose (Leguminosae), Vitex mollis H. B. K. (Verbenaceae), Ipomea bracteata Cav. (Convolvulaceae),
Bursera spp. (Burseraceae) y Cochlospermum vittifolium (Willd) Spreng (Cocholospermaceae) (Jardel, 1992). Los arroyos y cañones angostos del bosque se caracterizan por tener árboles más altos que aquellos presentes en el bosque tropical caducifolio.

Método de recolección. En diferentes sitios dentro del bosque se colocaron 6 trampas Malaise tipo Townes (Townes, 1972) y se mantuvieron en el mismo lugar durante todo el año. Estas trampas están diseñadas para atrapar insectos voladores, incluyendo microhimenópteros (Steyskal, 1981), y han demostrado ser un método eficiente de captura constante (Noyes, 1982; Kitching et al., 2001). Cada una de las trampas funcionó durante 5 días de cada mes. El líquido de preservación fue alcohol al 70\%. El trabajo de campo se realizó entre noviembre de 1996 y octubre de 1997. Los encírtidos de las localidades de Huautla (Morelos), Huatulco (Oaxaca) y San Javier (Sonora), todas con bosque tropical caducifolio, se estudiaron con la misma metodología que se utilizó en San Buenaventura, Jalisco, pero en años diferentes (Huautla: noviembre 1995-octubre 1996, Huatulco: febrero 2005-enero 2006, San Javier: noviembre 2003-octubre 2004).

Trabajo de laboratorio. El trabajo de laboratorio incluyó el procesamiento de 72 muestras ( 6 trampas $\times 12$ meses, agrupando como una sola muestra los ejemplares capturados durante los 5 días de recoleccción). Se separaron, secaron, montaron y etiquetaron los encírtidos contenidos en cada muestra. Antes de montar, los ejemplares fueron deshidratados, utilizando diferentes rangos de alcohol $(80 \%, 90 \%$ y absoluto por 24 horas cada uno) y acetato de amilo (2 horas) para evitar que colapsaran. La identificación de los encírtidos se obtuvo con ayuda de literatura especializada (Noyes, 1980, 2000; Noyes y Ren, 1995; Noyes et al., 1997) y mediante la comparación de ejemplares de colecciones científicas (TAMUIC, CNIN).

Métodos de análisis. Considerando que la riqueza de especies de una muestra generalmente subestima el valor de la riqueza real de especies en una localidad (Chazdon et al., 1998), se utilizó un método estadístico alternativo propuesto por Díaz-Francés y Gorostiza (Díaz-Francés y Soberón, 2005) para determinar cuan cerca se está de recolectar la riqueza real de los encírtidos potencialmente capturables con trampas Malaise en la región de estudio. Los modelos paramétricos utilizados fueron el logarítmico, el de Clench y el exponencial, que suponen que la probabilidad de encontrar una nueva especie depende del número de especies obtenidas y el tiempo que se invierte en trabajo de campo (Díaz-Francés y Soberón, 2005). Los modelos se ajustaron mediante procesos de "nacimiento puro" para estimar la riqueza de especies, utilizando el programa Species Accumulation Function (Centro de Investigación en Matemáticas, A.C., 2010), disponible en Internet en: http:// 
www.cimat.mx/index.php? $\mathrm{m}=266$. Para este procedimiento, las especies recolectadas en las 6 trampas Malaise por mes se consideraron como una sola muestra, habiéndose obtenido 12 muestras en total. El material estudiado se depositó en la colección entomológica del Instituto de Biología, UNAM (CNIN).

Los valores de riqueza y abundancia están representados por el número de especies e individuos recolectados. La diversidad se calculó con el índice de entropía de ShannonWiener $\left(\mathrm{H}^{`}\right)$. Este valor se calculó con el programa Bio Diversity Pro (McAlleece et al., 1999). Para el análisis fenológico se consideraron 2 estaciones, la lluviosa, que duró desde junio hasta noviembre, y la seca, que abarcó desde diciembre hasta mayo. Para entender el patrón de distribución local y temporal de las especies, se observó la diferencia en riqueza y abundancia de las especies en cada temporada. Para observar la similitud en abundancia y riqueza de especies entre las 4 localidades comparadas, se utilizaron 2 índices. Para comparar la abundancia se utilizó el de Sorensen cuantitativo que considera simultáneamente la abundancia en los sitios comparados y la abundancia relativa de cada especie en cada sitio. La riqueza de especies se comparó con el de similitud de Sorensen que utiliza datos de presencia/ausencia. Estos índices se calcularon con el programa Bio Diversity Pro (McAlleece et al., 1999).

\section{Resultados}

Riqueza. Durante el estudio, fueron recolectados 214 individuos de 61 especies de encírtidos; estas especies pertenecen a 30 géneros, 13 tribus y 2 subfamilias (Apéndice 1). La subfamilia con el mayor número de tribus, géneros y especies fue Encyrtinae, con 10 tribus (76.92\% del total), 22 géneros $(73.33 \%)$ y 44 especies
(72.13\%) (Cuadro 1). Las Tetracneminae presentaron 3 tribus $(23.07 \%), 8$ géneros $(26.66 \%)$ y 17 especies $(27.86 \%)$ (Cuadro 1). El género con el mayor número de especies recolectadas fue Metaphycus, con 11 especies (18\% del total), le siguen Anagyrus y Ooencyrtus con 7 (11\%), Aenasius y Cheiloneurus con 4 (6\%), Copidosoma con 3 (5\%), Adelencyrtus con 2 (3\%), y el resto de los géneros están representados por una sola especie (Apéndice 1).

Riqueza estimada. Con base en la razón de verosimilitud (rv), el modelo que mejor extrapoló los datos obtenidos fue el logarítmico ( $\mathrm{rv}=1)$; este modelo no es asintótico (Fig. 1). El modelo logarítmico es 1.417 veces más probable que el de Clench $(\mathrm{rv}=0.70)$, y 2.696 veces más probable que el exponencial ( $\mathrm{rv}=0.37)$. El número total de especies estimado para el modelo de Clench fue 801, y para el exponencial fue 16680 .

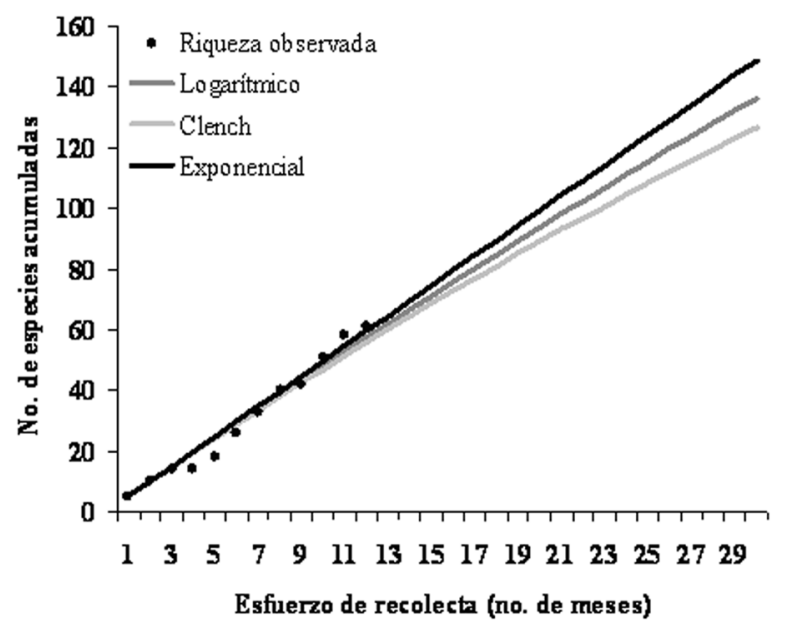

Figura 1. Curvas de acumulación de especies de la familia Encyrtidae en San Buenaventura.

Cuadro 1. Número y porcentaje de géneros, especies e individuos de las distintas tribus y subfamilias de Encyrtidae, recolectados con trampas Malaise en San Buenaventura

\begin{tabular}{llccc}
\hline Subfamilia & Tribu & Núm. géneros $y \%$ & Núm. especies $y \%$ & Núm. individuos $y \%$ \\
\hline Encyrtinae & Aphycini & $5(16.6 \%)$ & $15(24.59 \%)$ & $21(9.813 \%)$ \\
& Bothriothoracini & $1(3.33 \%)$ & $1(1.63 \%)$ & $2(0.934 \%)$ \\
Cheiloneurini & $3(10 \%)$ & $6(9.83 \%)$ & $8(3.738 \%)$ \\
Copidosomatini & $2(6.66 \%)$ & $4(6.55 \%)$ & $25(11.682 \%)$ \\
& Habrolepidini & $1(3.33 \%)$ & $2(3.27 \%)$ & $2(0.934 \%)$ \\
& Homalotylini & $2(6.66 \%)$ & $2(3.27 \%)$ & $2(0.934 \%)$ \\
Microteryni & $5(16.6 \%)$ & $11(18.03 \%)$ & $118(55.14 \%)$ \\
Metracneminae & $1(3.33 \%)$ & $1(1.63 \%)$ & $2(0.934 \%)$ \\
& Quadrencyrtini & $1(3.33 \%)$ & $1(1.63 \%)$ & $1(0.467 \%)$ \\
& Trechnitini & $1(3.33 \%)$ & $1(1.63 \%)$ & $1(0.467 \%)$ \\
Aenasiini & $3(10 \%)$ & $6(9.83 \%)$ & $6(2.80 \%)$ \\
Anagyrini & $3(10 \%)$ & $9(14.75 \%)$ & $24(11.214 \%)$ \\
& Tetracnemini & $2(6.66 \%)$ & $2(3.27 \%)$ & $2(0.934 \%)$ \\
& Trechnitini & $1(3.33 \%)$ & $1(1.63 \%)$ & $1(0.46 \%)$ \\
\hline
\end{tabular}


Abundancia. Las especies no fueron abundantes, ya que un $69 \%$ estuvo representado sólo por un individuo. La subfamilia más abundante fue Encyrtinae con 181 ejemplares, $84.5 \%$ del total; las Tetracneminae presentaron 33 individuos, 15.5\% (Cuadro 1). El género más abundante fue Ooencyrtus con 113 individuos (52.8\% del total), le siguió Copidosoma con 24 (11.21\%), Metaphycus con 17 (7.94\%), Pseudleptomastix con 14 (6.54\%) y Anagyrus con $9(4.20 \%)$, el resto de los géneros presentaron 6 o menos individuos. La especie más abundante fue Ooencyrtus pityocampae con 83 individuos (38.78\%), le siguió Ooencyrtus sp. 1 con 17 (7.94\%), Copidosoma sp. 2 con 15 (7\%), Pseudleptomastix sp.1 con $14(6.54 \%)$ y el resto de las especies están representadas por 5 individuos o menos.

Fenología. En general, la riqueza, abundancia y diversidad alcanzaron sus valores máximos en noviembre, durante la temporada de lluvias (Figs. 2, 3). El valor más bajo para estos parámetros se presentó en abril, durante la temporada de secas, en el que sólo se registraron 2 especies. Estacionalmente, 37 especies (60.6\% del total) estuvieron presentes sólo durante la época de lluvias; $9(14.7 \%)$ sólo durante la época de secas y $15(24.5 \%)$ en ambas temporadas (Cuadro 2). Durante la temporada de lluvias se registraron 129 individuos (60\% del total), y durante secas 85 (40\%). Se registraron 44 especies

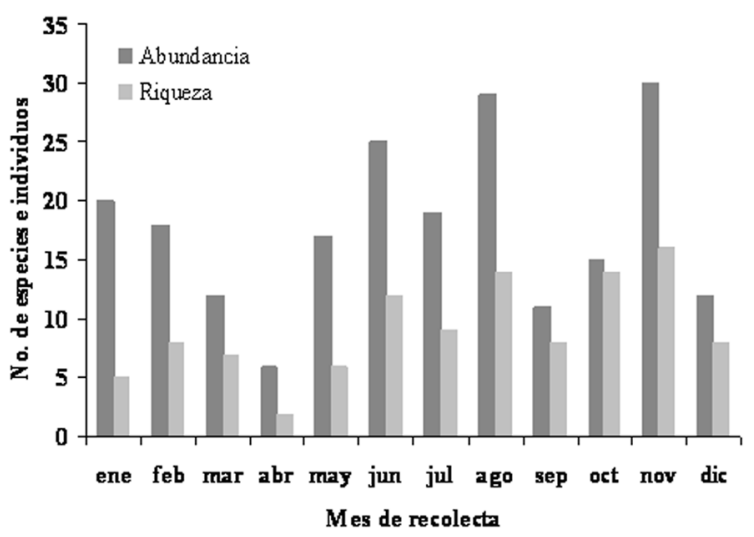

Figura 2. Riqueza y abundancia de los encírtidos recolectados en San Buenaventura.
(72.13\% del total) sólo en un mes, 7 (11.47\%) en 2 meses, $5(8.19 \%)$ durante 3 meses, $1(1.63 \%)$ durante 4 meses, $1(1.63 \%)$ durante 5 meses, $1(1.63 \%)$ durante 8 meses, $1(1.63 \%)$ durante 9 meses y $1(1.63 \%)$ durante 10 meses (Apéndice 1). Lo anterior indica que los adultos del $92 \%$ de las especies estuvieron activos por menos de 3 meses durante todo el año. De las 5 especies registradas como activas durante 4 meses o más, todas estuvieron presentes en ambas estaciones.

Comparación con otras regiones del occidente de México con bosque tropical caducifolio. El número de especies registradas en San Buenaventura (61) fue menor que el recolectado en Huautla (82) (Rodríguez-Velez y Woolley, 2005) y mayor que el obtenido en San Javier (52) (Rodríguez et al., en prensa) y Huatulco (17) (RodríguezVélez et al., 2009). De las especies recolectadas en San Buenaventura, 24 (39.34\% del total) no se registraron en ninguna de las otras 3 localidades; 5 especies $(8.19 \%)$ comparten las 3 localidades; 10 (16.39\%) comparte con San Javier y Huautla; $6(9.83 \%)$ con San Javier y 16 $(26.22 \%)$ con Huautla. De los 30 géneros recolectados en San Buenaventura, 10 (33.33\%) no se registran en ninguna otra localidad (Ageniaspis, Archinus, Cicoencyrtus, Eucoccidophagus, Meromyzobia, Mucrencyrtus, Pseudencyrtus, Pseudococcidae, Trechnites y Zaomma), $5(16.66 \%)$ géneros comparten las 3 localidades (Ana-

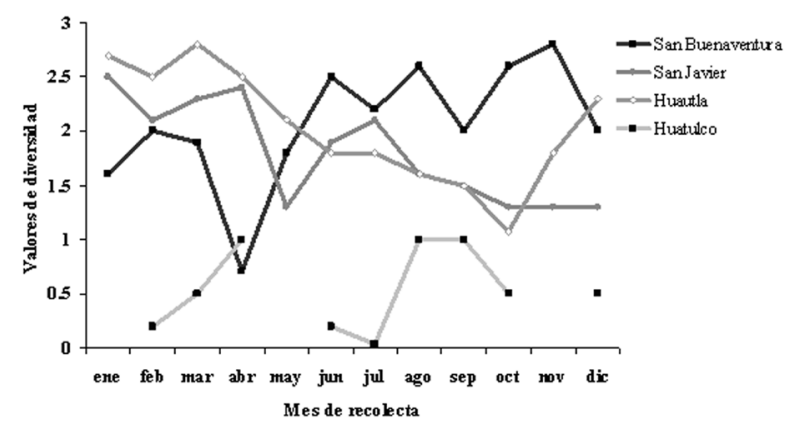

Figura 3. Diversidad calculada con el índice de entropía de Shannon-Wiener (H') para encírtidos de 4 localidades con bosque tropical caducifolio del occidente de México.

Cuadro 2. Porcentaje del número de especies y abundancia estacional de la familia Encyrtidae en 4 localidades con bosque tropical caducifolio del occidente de México

\begin{tabular}{lccccc}
\hline & \multicolumn{2}{c}{ Estación de lluvias } & Estación de secas & \multicolumn{2}{c}{ Ambas estaciones } \\
Localidades & Núm. especies & Abundancia & Núm. especies & Abundancia & Núm. especies \\
\hline San Buen & $61 \%$ & $60 \%$ & $15 \%$ & $40 \%$ & $24 \%$ \\
San Javier & $27 \%$ & $30 \%$ & $54 \%$ & $70 \%$ & $19 \%$ \\
Huautla & $36 \%$ & $55 \%$ & $43 \%$ & $45 \%$ & $21 \%$ \\
Huatulco & $47 \%$ & $50 \%$ & $47 \%$ & $50 \%$ & $6 \%$ \\
\hline
\end{tabular}


gyrus, Metaphycus y Ooencyrtus, Pseudleptomastix, Rhytidhothorax), 5 (16.66\%) con San Javier y Huautla (Aenasius, Cheiloneurus, Epanusia, Holcencyrtus y Prochiloneurus), 2 (6.66\%) con San Javier (Syrphophagus y Tetracnemus) y 7 (23.33\%) con Huautla (Agarwalencyrtus, Blepyrus, Copidosoma, Discodes, Homalotyloidea, Homalotylus y Zarhopalus).

Los valores más altos del índice de diversidad se presentaron durante la estación seca para las localidades de San Javier y Huautla, mientras que la localidad de Huatulco mostró valores altos en ambas temporadas. Sin embargo, la diversidad de San Buenaventura fue mayor durante la temporada de lluvias (Fig. 3). El número de especies fue más alto durante la estación seca para las localidades de San Javier y Huautla. Huatulco mostró el mismo valor de riqueza en ambas temporadas (Cuadro 2), y nuevamente San Buenaventura presenta una mayor riqueza para la temporada de lluvias. Huatulco no mostró diferencia estacional en abundancia, San Javier fue más abundante en secas, y Huautla y San Buenaventura durante la época de lluvias (Cuadro 2). El 67\% de las especies de San Javier, el $88 \%$ de las de Huatulco, el $71 \%$ de Huautla y el $72 \%$ de San Buenaventura, sólo se encuentran activas durante un mes. El $67 \%$ de las especies de San Javier, el 88\% de Huatulco, el 65\% de Huautla y el 79\% de San Buenaventura están representadas sólo por un individuo. San Buenaventura y Huautla son las localidades más similares en cuanto a riqueza y abundancia, mostrando un índice de similitud de 43.97 y 52.83, respectivamente (Cuadro 3). San Buenaventura mostró menos similitud con Huatulco (Cuadro 3).

\section{Discusión}

De acuerdo con la riqueza de especies registrada en San Buenaventura, Jalisco, y los valores de la riqueza estimada que se obtuvieron con los modelos paramétricos, es probable que la riqueza de encírtidos que se observa sea mucho menor que el verdadero número de especies presentes en la zona. Los resultados de los modelos paramétricos sugieren que el modelo logarítmico es el que mejor extrapola los datos. Este modelo no es asintótico, por lo tanto, no sugiere un posible número de especies en el área. En estudios, este modelo se ajusta en su primer fase, cuando la región estudiada es muy grande y/o los taxones son poco conocidos (Díaz-Francés y Gorostiza, 2002). El estudio de los encírtidos en San Buenaventura se ajusta a estos supuestos (zona grande, su fauna es poco conocida y es el único estudio en el que se ha recolectado encírtidos de una manera sistemática en la región).

El aparente bajo número de especies registradas puede reflejar las limitaciones de la técnica de muestreo, pero también puede deberse a la biología del grupo examinado. Es muy probable que las especies raras de encírtidos con períodos cortos de vida no se hayan recolectado porque su actividad como adultos no coincidió con los días en que se recolectó (5 días por mes). La aparente rareza de algunas especies, aunada con sus periodos cortos de actividad y la heterogeneidad del BTC -las características de los suelos, por ejemplo, con su variación topográfica-, que causa cambios en la composición florística (Trejo, 1998), disminuyen la probabilidad de recolectar especies que sólo aparecen en hábitats específicos o que están estrechamente ligadas a recursos escasos en el ecosistema, tales como huéspedes o plantas.

Hasta el momento se ha registrado un comportamiento fenológico diferente entre localidades. La riqueza de especies de San Javier y Huautla muestra una marcada estacionalidad, siendo mayor durante la estación seca, a diferencia de Huatulco que no mostró un patrón estacional. No obstante, la riqueza de especies de San Buenaventura tuvo una marcada estacionalidad, siendo la temporada de lluvias la más rica y abundante. Posiblemente esta diferencia fenológica corresponde a la gran heterogeneidad que presenta la selva baja caducifolia, tanto en una misma zona como en diferentes regiones; sin embargo, pudo deberse a que cada región se estudió en diferentes años, lo cual puede haber afectado diferentes condiciones ambientales influyendo, así, a las poblaciones de encírtidos.

Según el índice de similitud, las localidades con mayor afinidad en cuanto a riqueza y abundancia son San Buenaventura y Huautla (Cuadro 3). En ambas hay más actividad humana, lo cual podría formar una mayor varie-

Cuadro 3. Valores de similitud faunística de abundancia (A) y riqueza (R) obtenidos con los índices de Sorensen cuantitativo y similitud de Sorensen para los encírtidos recolectados en 4 localidades con bosque tropical caducifolio en el occidente de México

\begin{tabular}{lcccccccc}
\hline & \multicolumn{2}{c}{ San Javier } & \multicolumn{2}{c}{ Huautla } & \multicolumn{2}{c}{ Huatulco } & \multicolumn{2}{c}{ San Buenaventura } \\
& $A$ & $R$ & $A$ & $R$ & $A$ & $R$ & $A$ & $R$ \\
\hline San Javier & $*$ & $*$ & 35.99 & 40.31 & 16.35 & 29.50 & 35.95 & 38.18 \\
Huautla & $*$ & $*$ & $*$ & $*$ & 8.28 & 17.39 & 52.83 & 43.97 \\
Huatulco & $*$ & $*$ & $*$ & $*$ & $*$ & $*$ & 7.79 & 13.69 \\
San Buenaventura & $*$ & $*$ & $*$ & $*$ & $*$ & $*$ & $*$ \\
\hline
\end{tabular}


dad de microhábitats, tales como agroecosistemas, que pueden servir como refugio y fuente de alimento para una gran variedad de insectos.

\section{Agradecimientos}

Al Posgrado de Ciencias Biológicas (UNAM), al Instituto de Biología (UNAM) y al Consejo Nacional de Ciencia y Tecnología (CONACYT) por el apoyo recibido para la realización de este proyecto.

\section{Literatura citada}

Ceballos, G. y A. García. 1995. Conserving Neotropical biodiversity: The role of dry forests in western Mexico. Conservation Biology 9:1349-356.

Centro de Investigación en Matemáticas, A.C. 2010. Species Accumulation. http://www.cimat.mx/index.php?m=266; última consulta: 10.VI.2010.

Chazdon, R. L., R. K. Colwell, J. S. Denslow y M. R. Guariguata. 1998. Statistical methods for estimating species richness of woody regeneration in primary and secondary rain forest of northeastern Costa Rica. In Forest biodiversity research, monitoring and modeling, conceptual background and Old World case studies, F. Dallmeier y J. A. Comiskey (eds.). Man and the Biosphere Series, Paris. p. 285-309.

CNA (Comisión Nacional del Agua). 2000. Datos climatológicos de la presa B. Badillo, municipio El Limón, Jalisco. Comisión Nacional del Agua, Jalisco, México.

Díaz-Francés, E. y L. G. Gorostiza. 2002. Inference and model comparison for species accumulation functions using approximating pure birth processes. Journal of Agricultural, Biological, and Environmental Statistics 7:335-349.

Díaz-Francés, E. y J. Soberón. 2005. Statistical estimation and model selection of species-accumulation functions. Conservation Biology 19:569-573.

García, E. 1981. Modificaciones al sistema de clasificación climática de Köppen. Instituto de Geografía, UNAM, México, D. F. 246 p.

Janzen, D. H. 1988. Tropical dry forest: The most endangered major tropical ecosystem. In Biodiversity, E. O. Wilson (ed.). National Academy Press, Washington, D. C. p. 130-137.

Jardel, E. J. P. (Coord.). 1992. Estrategia para la conservación de la Reserva de la Biosfera Sierra de Manantlán. Editorial Universidad de Guadalajara, Jalisco. 315 p.

Kitching, R. L., D. Li y N. E. Stork. 2001. Assessing biodiversity 'sampling packages': How similar are arthropod assemblages in different tropical rainforest? Biodiversity and Conservation 10:793-813.

McAleece, N., P. J. D. Lambshead y J. D. Cage. 2010. Biodiversity pro.user's guide and application. http:/www.sams.ac.uk/ research/software/software; última consulta: 10.VI.2010.
Millennium Ecology Assessment. 2005. Ecosystems and human well-being: biodiversity synthesis. World Resources Institute, Washington, D. C. 100 p.

Noyes, J. S. 1980. A review of the genera of Neotropical Encyrtidae (Hymenoptera: Chalcidoidea). Bulletin of the British Museum, Natural History 41:107-253.

Noyes, J. S. 1982. Collecting and preserving chalcid wasps (Hymenoptera: Chalcidoidea). Journal of Natural History 16:315-334.

Noyes, J. S. 1988. Encyrtidae (Insecta: Hymenoptera). Fauna of New Zealand. 192 p.

Noyes, J. S. 1989. The diversity of Hymenoptera in the tropics, with special reference to Parasitica in Sulawesi. Ecological Entomology 14:97-207.

Noyes, J. S. 2000. Encyrtidae of Costa Rica (Hymenoptera: Chalcidoidea), 1. The subfamily Tetracneminae, parasitoids of mealybugs (Homoptera: Pseudococcidae). Memoirs of the American Entomological Institute 62:1-355.

Noyes, J. S. y H. Ren. 1995. Encyrtidae of Costa Rica (Hymenoptera: Chalcidoidea): the genus Aenasius Walker, parasitoids of mealybugs (Homoptera: Pseudococcidae). Bulletin of The Natural History Museum (Entomology Series) 64:117-163.

Noyes, J. S., J. B. Woolley y G. Zolnerowich. 1997. Family Encyrtidae. In Annotated keys to the genera of Nearctic Chalcidoidea (Hymenoptera), G. A. P. Gibson, J. T. Huber y J. B. Woolley (eds.). National Research Council Canada, NRC, Ottawa. p. 70-320.

Rodríguez, J. M., B. Rodríguez-Velez, S. Zaragoza-Caballero, F. A. Noguera, E. González-Soriano y E. Ramírez-García. Diversity of Encyrtidae (Hymenoptera: Chalcidoidea) collected with Malaise traps in the tropical dry forest of San Javier, Sonora, Mexico. Revista Mexicana de Biodiversidad (en prensa).

Rodríguez-Velez, B. y J. B. Woolley. 2005. La fauna de la familia Encyrtidae (Hymenoptera: Chalcidoidea) en el bosque tropical caducifolio de la sierra de Huautla, Morelos, México. Folia Entomológica Mexicana 44:147-155.

Rodríguez-Velez, B., S. Zaragoza-Caballero y J. M. Rodríguez. 2009. Diversidad de Encyrtidae (Hymenoptera: Chalcidoidea) y otras familias de Hymenoptera obtenidas con trampas Malaise en el bosque tropical caducifolio de la región de Huatulco, Oaxaca, México. Revista Mexicana de Biodiversidad 80:709-719.

Steyskal, G. C. 1981. A bibliography of the malaise trap. Proceedings of the Entomological Society of Washington 83:225-229.

Townes, H. 1972. A light-weight Malaise trap. Entomological News 83:239-247.

Trejo, I. 1998. Distribución y diversidad de selvas bajas de México: Relaciones con el clima y el suelo. Tesis, Facultad de Ciencias, Universidad Nacional Autónoma de México. México D. F. 210 p. 
Trejo, I. 2010. Las selvas secas del Pacífico mexicano. In Diversidad, amenazas y áreas prioritarias para la conservación de las selvas secas del Pacífico de México, G. Ceballos, L. Martínez, A. García, E. Espinoza, J. Bezaury Creel y R. Dirzo (eds.). México. D.F. p. 41-51.
Wilson, E. O. 1988. The current state of biological diversity. In Biodiversity, E. O. Wilson (ed.). National Academic Press, Washington, D. C. p. 3-18.

Wolda, H. 1983. Diversity, diversity indices and tropical cockroaches. Oecologia 58:290-298.

Apéndice 1. Lista de los encírtidos recolectados con trampas Malaise en el bosque tropical caducifolio de San Buenaventura, Jalisco, México; seguido de la especie se menciona el número de individuos recolectados y en números romanos el mes de recolecta.

\begin{tabular}{|c|c|}
\hline Subfamilia & Homalotyloidea sp. 1, 1, XII \\
\hline Encyrtinae & Homalotylus brevicauda, $1, \mathrm{X}$ \\
\hline Tribu & Tribu \\
\hline Aphycini & Microteryini \\
\hline Especies & Especies \\
\hline Archinus cupatus, $1, \mathrm{~V}$ & Ooencyrtus pityocampae, 83, I, II, III, V, VI, VII, VIII, IX, XI, \\
\hline Cicoencyrtus sp.1, 1, I & XII \\
\hline Metaphycus sp. 1, 1, VII & Ooencyrtus sp. 1, 17, I, II, IV, VI, VII, VIII, IX, X, XI \\
\hline Metaphycus sp. 2, 3, VIII, IX, XI & Ooencyrtus sp. $2,1, \mathrm{X}$ \\
\hline Metaphycus sp. 3, 1, VII & Ooencyrtus sp. 3, 2, III, VIII \\
\hline Metaphycus sp. 4, 2, V, XI & Ooencyrtus sp. 4, 5, III, VI, VIII, IX \\
\hline Metaphycus sp. 5, 1, XI & Ooencyrtus sp. 5, 3, VIII, XI, XII \\
\hline Metaphycus sp. 6, 2, VI, XI & Ooencyrtus sp. 6, 2, II, VI \\
\hline Metaphycus sp. 7, 3, XI & Agarwalencyrtus citri, 1, XI \\
\hline Metaphycus sp. 8, 1, VIII & Mucrencyrtus sp. 1, 2, VIII \\
\hline Metaphycus sp. 9, 1, VII & Pseudencyrtus sp. 1, 1, VII \\
\hline Metaphycus sp. 10, 1, XI & Syrphophagus sp. 1, 1, X \\
\hline Metaphycus sp. 11, 1, XI & Tribu \\
\hline Pseudaphycus flavidulus, 1, III & Miraini \\
\hline Pseudococcobius sp. 1, 1, X & Especies \\
\hline Tribu & Meromyzobia sp. 1, 2, VI, IX \\
\hline Bothriothoracini & Tribu \\
\hline Especies & Quadrencyrtini \\
\hline Rhytidothorax sp. 1, 2, II, VIII & Especies \\
\hline Tribu & Eucoccidophagus sp. 1, 1, VI \\
\hline Cheiloneurini & Tribu \\
\hline Especies & Trechnitini \\
\hline Cheiloneurus claviger, 3, I, II, XI & Especies \\
\hline Cheiloneurus elegans, 1, VII & Trechnites sp. 1, 1, V \\
\hline Cheiloneurus sp. 1, 1, X & Subfamilia \\
\hline Cheiloneurus sp. 2, 1, VI & Tetracneminae \\
\hline Prochiloneurus bolivari, $1, \mathrm{XI}$ & Tribu \\
\hline Zaomma sp. 1, 1, VII & Aenasiini \\
\hline Tribu & Especies \\
\hline Copidosomatini & Aenasius advena, $1, \mathrm{I}$ \\
\hline Especies & Aenasius sp. 1, 1, XII \\
\hline Ageniaspis citricola, $1, \mathrm{X}$ & Aenasius sp. 2, 1, XI \\
\hline Copidosoma floridanum, 4 & Aenasius sp. $3,1, \mathrm{X}$ \\
\hline Copidosoma sp. 1, 5, VIII, X, XII & Blepyrus sp. 1, 1, VI \\
\hline Copidosoma sp. 2,15 & Holcencyrtus sp. 1, 1, VII \\
\hline Tribu & Tribu \\
\hline Habrolepidini & Anagyrini \\
\hline Especies & Especies \\
\hline Adelencyrtus odonaspidis, 1, XII & Anagyrus lopezi, 3, VI, VIII, XII \\
\hline Adelencyrtus sp. $1,1, \mathrm{X}$ & Anagyrus paralia, $1, \mathrm{X}$ \\
\hline Tribu & Anagyrus pseudococci, 1, IX \\
\hline Homalotylini & Anagyrus sp. 1, 1, III \\
\hline Especies & Anagyrus sp. 2, 1, X \\
\hline
\end{tabular}


Apéndice 1. Continúa.

Anagyrus sp. 3, 1, II

Anagyrus sp. 4, 1, VI

Epanusia sp. 1, 1, VI

Pseudleptomastix sp. 1, 14, II, III, V, VIII, IX, X, XI, XII
Tribu

Tetracnemini

Especies

Tetracnemus sp. 1, 1, VIII

Zarhopalus sp. 1, 1, VIII 